\title{
On the Number of Facets of Three-Dimensional Dirichlet Stereohedra I: Groups with Reflections*
}

\author{
D. Bochiş and F. Santos
}

Departamento de Matemáticas, Estadística y Computación, Universidad de Cantabria, Santander, Spain

\{dacib, santos\}@matesco.unican.es

\begin{abstract}
A Dirichlet stereohedron for a crystallographic group $G$ is the Voronoi region of any point with trivial stabilizer in the Voronoi diagram of its orbit.

We prove that Dirichlet stereohedra for three-dimensional crystallographic groups containing reflections in three, two or one independent directions cannot have more than eight, eighteen and fifteen facets, respectively. We show examples where the three bounds are attained.

As a tool for one of the cases, we study how many regions of a Dirichlet tesselation in the plane can be intersected by each region of another Dirichlet tesselation for the same group. We find out that the number is at most seven for all planar crystallographic groups except, perhaps, those of type pgg.
\end{abstract}

\section{Introduction}

This paper deals with the combinatorial types of Dirichlet stereohedra in three dimensions. More precisely, we find the exact maximum number of facets of three-dimensional Dirichlet stereohedra corresponding to groups which contain reflections.

A discrete group of motions in $\mathbb{R}^{d}$ is a group of Euclidean isometries whose orbits are discrete. If it contains $d$-independent translations, then it is a crystallographic group. Equivalently, a discrete group $G$ is crystallographic if it admits a compact fundamental domain. A fundamental domain for $G$ is any closed connected region with non-empty interior and which covers $\mathbb{R}^{d}$ by the action of $G$ without gaps or overlaps.

* The second author was partially supported by Grant PB97-0358 of the Spanish Dirección General de Investigación Científica y Técnica. 
A special manner to find fundamental domains for a crystallographic group is to compute the Voronoi diagram of the orbit of a point with trivial stabilizer. The (closed) regions in the Voronoi diagram are fundamental domains and they are called Dirichlet domains. In the theory of tessellations, a stereohedron is any bounded convex polyhedron which tiles the space by the action of a crystallographic group. Dirichlet domains are stereohedra and, hence, they are also called Dirichlet stereohedra.

The study of the combinatorial types of stereohedra is related to Hilbert's 18th problem, "Building up the space with congruent polyhedra" (see [11] and [15]). Although Bieberbach (1910) and Reinhardt (1932) gave complete answers to the first two of Hilbert's specific questions, other problems related to monohedral tessellations (i.e. tessellations whose tiles are congruent, such as those which appear in Hilbert's 18th problem) remain open. An exhaustive account of them appeared in a survey article by Grünbaum and Shephard [9]. In particular, they ask one to determine the maximum number of $(d-1)$-dimensional faces (i.e. facets)-or, at least, a "good" upper bound-of convex polyhedral tiles of monohedral tessellations in $\mathbb{R}^{d}$. This problem, for the case of stereohedral tessellations, is also mentioned in [18].

The only general result known concerning this is the fundamental theorem of stereohedra proved by Delone in 1961 [6]. It says that a stereohedron associated to a crystallographic group with $a$ aspects in $\mathbb{R}^{d}$ has at most $2^{d}(a+1)-2$ facets. Here, the number of aspects of a crystallographic group $G$ is the index $\left[G: G_{T}\right]$ of its translational subgroup $G_{T}$. In other words, the number of translational lattices in which a generic orbit of $G$ decomposes. As a corollary to our analysis of general properties of stereohedra, in Section 1 we provide a proof of Delone's theorem for the case of Dirichlet stereohedra (Theorem 1.8).

Starting with Fedorov, who studied some Dirichlet stereohedra with 14 facets in 1899 , there have been several attempts to find three-dimensional stereohedra with "many" facets. Föppl, Novacki and Smith discovered Dirichlet stereohedra with 16, 18 and 20 facets, respectively, in 1916, 1935 and 1965 [8], [16], [19]. In 1972 Koch [12] found Dirichlet stereohedra with 23 facets and later together with Fischer [13] he produced some with 24 facets. With the data available at that time and some heuristic arguments, in 1978 Brunner and Laves conjectured 26 to be an upper bound for the number of facets of three-dimensional Dirichlet stereohedra and $3^{d}-1$ for the $d$-dimensional case [4].

This conjecture was found to be false 2 years later when Engel, with computer-assisted techniques, found several families of Dirichlet stereohedra with facets from 17 to 38 , for a cubic group with 24 aspects (see [7]). These are the stereohedra with the maximum number of facets known so far, while Delone's upper bound in three dimensions is 390 since the maximum number of aspects of a three-dimensional crystallographic group is 48. There is agreement among the experts that the maximum number of facets that a stereohedron can attain is closer to 38 than to 390 (see [7, page 214], [9, page 960] and [18, page 50]).

Observe that in the constructions mentioned above only the particular case of Dirichlet stereohedra (also known as plesiohedra) is considered even if, as observed by Grünbaum and Shephard [9, page 965], "there seems to be no grounds to assume that all stereohedra are combinatorially equivalent to plesiohedra (though no example settling this question is known)." The reason for this restriction is the absence of practical methods for constructing non-Dirichlet stereohedra. As the same authors state, "there seems to be no 
practical technique available which would be guaranteed to produce-if only carried out with sufficient perseverance-all combinatorial types of stereohedra". Algorithms for the same task restricted to Dirichlet stereohedra are easy to design, since the existence of a particular combinatorial type for a particular affine equivalence class of crystallographic groups is a quantifier elimination problem over the reals. However, the "perseverance" needed to accomplish this task seems beyond the capabilities of present computers.

In this paper and the subsequent ones [2] and [3] we intend to find a more realistic upper bound for the maximum number of facets of a three-dimensional Dirichlet stereohedron. The results we have obtained so far are: For groups with reflections, an optimal upper bound of 18 facets (this paper). For groups without reflections, upper bounds of 102 for non-cubic groups and 162 for the cubic ones (these can be found in [1]).

The method we use for studying Dirichlet stereohedra for a group $G$ with reflections is based on the use of "reflection cells" of $G$, defined as the fundamental domains of the reflection subgroup of $G$ (or, equivalently, the regions in which the reflection planes of $G$ divide the space). Every stereohedron for $G$ is contained in a reflection cell (see Proposition 2.2). We first consider the number of facets that a Dirichlet stereohedron can have inside and on the boundary of the reflection cell, obtaining bounds of 16 and 6 . Studying the dependence between the two types of facets, we get a global bound of 18 .

Actually, we study separately the cases of groups having reflections in three, two or one independent directions (Sections 2.1-2.3). We get upper bounds of 8,18 and 15 facets respectively (Theorems $2.4,2.7$ and 2.12) and examples where the three bounds are attained (Examples 2.5, 2.9 and 2.13). Summing up, our main result is:

Theorem. The exact maximum number of facets of a three-dimensional Dirichlet stereohedron corresponding to a group with reflections is 18 .

When studying the case of reflections in only one direction in Section 2.3 we will need to address the following problem: given two orbits $G P$ and $G Q$ of the same planar crystallographic group, how many regions of $\operatorname{Vor}_{G P}(P)$ can intersect each region of $\operatorname{Vor}_{G Q}(Q)$ ? Section 3 is devoted to studying this problem. We prove that the number of regions is at most seven in all groups except perhaps those of type $p g g$, and that there is an explicit example where seven is attained, for the group $p 2$. For $p g g$, we can only prove the bound of seven under the assumption that $G P \cup G Q$ is again an orbit of a crystallographic group, which is the case needed in Section 2.3.

Throughout the paper we use the International Crystallographic notation for planar and three-dimensional crystallographic groups; see, e.g. [14] and [17].

\section{Some General Properties of Dirichlet Stereohedra}

For any discrete set of points $S \subset \mathbb{R}^{d}$, the (open) Voronoi region corresponding to a point $P \in S$, denoted $\operatorname{Vor}_{S}(P)$, is the region of $\mathbb{R}^{d}$ containing those points which are closer to $P$ than to any other point of $S$, i.e.

$$
\operatorname{Vor}_{S}(P)=\left\{x \in \mathbb{R}^{d}: d(x, P)<d(x, Q), \forall Q \in S \backslash\{P\}\right\} .
$$


The closure of $\operatorname{Vor}_{S}(P)$ is a convex polyhedron and it can be described as

$$
\overline{\operatorname{Vor}_{S}(P)}=\left\{x \in \mathbb{R}^{d}: d(x, P) \leq d(x, Q), \forall Q \in S \backslash\{P\}\right\}
$$

Two points $P, Q \in S$ are called neighbours in the Voronoi diagram $\operatorname{Vor}_{S}$ if $\overline{\operatorname{Vor}_{S}(P)}$ and $\overline{\operatorname{Vor}_{S}(Q)}$ share a facet. Equivalently, if there exists a sphere $\mathcal{C}$ which passes through $P$ and $Q$ and which does not contain any other point of $S$. Here are some straightforward properties of Voronoi diagrams that we use:

Lemma 1.1. Let $S$ be a discrete set of points in $\mathbb{R}^{d}$.

(i) Let $S$ be partitioned in two parts $S_{1}$ and $S_{2}$ (i.e $S_{1} \cap S_{2}=\emptyset$ and $S=S_{1} \cup S_{2}$ ). If $P \in S_{1}$ and $Q \in S_{2}$ are neighbours in $\operatorname{Vor}_{S}$, then $\operatorname{Vor}_{S_{1}}(P) \cap \operatorname{Vor}_{S_{2}}(Q) \neq \emptyset$.

(ii) If $S^{\prime} \subseteq S$ and $P \in S$, then $\operatorname{Vor}_{S}(P) \subseteq \operatorname{Vor}_{S^{\prime}}(P)$.

(iii) If $A, B, C, D \in S$ form a planar quadrilateral with vertices in that order, then

$$
\angle A+\angle C \leq 180^{\circ} \Rightarrow A \text { is not a neighbour of } C \text { in Vors } .
$$

The Voronoi diagram of an orbit of a crystallographic group is called a Dirichlet tessellation. Throughout the rest of this section $G$ is a crystallographic group (of arbitrary dimension $d$ ) and $G P$ is the orbit of a point $P$ with trivial stabilizer. We are interested in the neighbours of $P$ in $\operatorname{Vor}_{G P}$. Each such neighbour corresponds to a facet of the Dirichlet stereohedron $\operatorname{Vor}_{G P}(P)$. The following result implies that, for any $g \in G, g P$ is a neighbour of $P$ if and only if $g^{-1} P$ is also a neighbour.

Proposition 1.2. Let $g \in G$. If $f$ is the common facet of $\operatorname{Vor}_{G P}(P)$ and $\operatorname{Vor}_{G P}(g P)$, then $g^{-1} f$ is the common facet of $\operatorname{Vor}_{G P}(P)$ and $\operatorname{Vor}_{G P}\left(g^{-1} P\right)$.

Our methods for bounding the number of neighbours of $P$ in $\operatorname{Vor}_{G P}$ are based in decomposing $G P$ via parallel subspaces (lines or planes) and bounding the number of neighbours in each of them separately.

Lemma 1.3. Let $\alpha$ be an affine subspace and let $\beta \neq \alpha$ be a translate of $\alpha$; let $S_{\alpha}=G P \cap \alpha$ and $S_{\beta}=G P \cap \beta$. Let $\pi_{\alpha}: \mathbb{R}^{d} \rightarrow \alpha$ denote the orthogonal projection to $\alpha$ and let $S_{\alpha}^{\prime}:=\pi_{\alpha}\left(S_{\beta}\right)$. Let $P \in \alpha$ and $Q \in \beta$ be neighbours in Vor $V_{G}$. Then $\operatorname{Vor}_{S_{\alpha}}(P) \cap \operatorname{Vor}_{S_{\alpha}^{\prime}}\left(\pi_{\alpha} Q\right) \neq \emptyset$.

Proof. Observe that $S_{\beta}$ and $S_{\alpha}$ are disjoint. Hence, $\operatorname{Vor}_{S_{\beta}}(Q) \cap \operatorname{Vor}_{S_{\alpha}}(P) \neq \emptyset$ by parts (i) and (ii) of Lemma 1.1.

Let $V$ denote the linear subspace orthogonal to $\alpha$ and $\beta$. Then $\operatorname{Vor}_{S_{\alpha}}(P)=\left(\operatorname{Vor}_{S_{\alpha}}(P) \cap\right.$ $\alpha)+V$ and $\operatorname{Vor}_{S_{\beta}}(Q)=\left(\operatorname{Vor}_{S_{\beta}}(Q) \cap \beta\right)+V=\operatorname{Vor}_{S_{\alpha}^{\prime}}\left(\pi_{\alpha} Q\right)$. This finishes the proof.

Lemma 1.4. Let $G_{T}$ be a crystallographic group generated by dinearly independent translations and $P \in \mathbb{R}^{d}$. Let $G_{T}^{2}=\left\{t^{2}: t \in G_{T}\right\}$ be the subgroup of $G_{T}$ generated by 
translations of vectors double the vectors of $G_{T}$. Then the following equalities hold:

$$
\begin{aligned}
\left\{Q \in \mathbb{R}^{d}:\right. & \left.\operatorname{Vor}_{G_{T} P}(P) \cap \operatorname{Vor}_{G_{r} Q}(Q) \neq \emptyset\right\} \\
& \stackrel{(1)}{=}\left\{Q \in \mathbb{R}^{d:}: \frac{P+Q}{2} \in \operatorname{Vor}_{G_{T} P}(P) \cap \operatorname{Vor}_{G_{T} Q}(Q)\right\} \\
& \stackrel{(2)}{=}\left\{Q \in \mathbb{R}^{d}: \frac{P+Q}{2} \in \operatorname{Vor}_{G_{T} P}(P)\right\} \\
& \stackrel{(3)}{=} \operatorname{Vor}_{G_{T}^{2} P}(P) .
\end{aligned}
$$

Proof. Let $i_{P}$ be the inversion with centre $P\left(i_{P}^{2}\right.$ equals the identity and $P$ is the unique fixed point of $\left.i_{P}\right)$. Then $i_{P} G_{T} P=G_{T} P$ and therefore $\operatorname{Vor}_{G_{T} P}(P)=i_{P} \operatorname{Vor}_{G_{T} P}(P)=$ $i(P+Q) / 2\left(t_{P Q} \operatorname{Vor}_{G_{T}}(P)\right)$, where $t_{P Q}$ is the translation of vector $P Q$. Since $t_{P Q}$ $\operatorname{Vor}_{G_{r} P}(P)=\operatorname{Vor}_{G_{T} Q}(Q)$, we have $\operatorname{Vor}_{G_{T} P}(P)=i(P+Q) / 2 \operatorname{Vor}_{G_{T} Q}(Q)$. If the intersection $\operatorname{Vor}_{G_{T} P}(P) \cap \operatorname{Vor}_{G_{T} Q}(Q)$ is not empty, since it is convex, the point $(P+Q) / 2$ has to belong to it and we are done with (1).

For the equality (2), it suffices to prove that $(P+Q) / 2$ is in $\operatorname{Vor}_{G P}(P)$ if and only if $(P+Q) / 2$ is in $\operatorname{Vor}_{G Q}(Q)$. This holds since

$$
\begin{aligned}
d\left(\frac{P+Q}{2}, P\right) \leq d\left(\frac{P+Q}{2}, t P\right), \forall t \in G_{T} \\
\Leftrightarrow \quad d\left(\frac{P+Q}{2}, Q\right) \leq d\left(\frac{P+Q}{2},-t Q\right), \forall t \in G_{T} \\
\Leftrightarrow \quad d\left(\frac{P+Q}{2}, Q\right) \leq d\left(\frac{P+Q}{2}, t Q\right), \forall t \in G_{T} .
\end{aligned}
$$

For (3), let $Q$ be such that

$$
\begin{aligned}
\frac{P+Q}{2} \in \operatorname{Vor}_{G_{T} P}(P) \Leftrightarrow d\left(\frac{P+Q}{2}, P\right) \leq d\left(\frac{P+Q}{2}, t P\right) \\
\Leftrightarrow \quad d(P, Q) \leq d(Q, 2 t P), \forall t \in G_{T} \Leftrightarrow Q \in \operatorname{Vor}_{G_{T}^{2} P}(P) .
\end{aligned}
$$

Note that the Voronoi region $\operatorname{Vor}_{G_{T}^{2}}(P)$ is the dilation of factor 2 of the region $\operatorname{Vor}_{G_{T}}(P)$ and therefore the open Voronoi region $\operatorname{Vor}_{G_{T}^{2}}(P)$ contains at most $2^{d}$ points of any orbit $G Q$ of $G$. With this and Lemma 1.4 we have:

Lemma 1.5. Let $G_{T}$ be a crystallographic group generated by dinearly independent translations and let $P, Q \in \mathbb{R}^{d}$. Then $\operatorname{Vor}_{G_{T} P}(P)$ intersects at most $2^{d}$ regions of $\operatorname{Vor}_{G_{T}} Q$.

Corollary 1.6. Let $G$ be a crystallographic group with a aspects in $\mathbb{R}^{d}$. Let $G P$ and $G Q$ be two different orbits. Then the Voronoi region $\operatorname{Vor}_{G P}(P)$ intersects at most $2^{d} a$ Voronoi regions of $\operatorname{Vor}_{G Q}$.

Proposition 1.7. Let $G$ be a crystallographic group. Let $\alpha$ be an affine subspace of $\mathbb{R}^{d}$ of a certain dimension $k$. Let $G_{0}$ be the subgroup of $G$ which fixes all the affine subspaces that are translates of $\alpha$, i.e. $G_{0}=\{g \in G: g(t \alpha)=t \alpha, \forall$ translation $t\}$. 
Suppose moreover that $G_{0}$ is a crystallographic group, regarded as a group of motions in $\alpha$ (i.e., that $G_{0}$ contains $k$ independent translations).

Let $P \in \alpha$ be a point with trivial stabilizer under $G$ and let $\beta \neq \alpha$ be a translate of $\alpha$. Then $P$ has at most $2^{k} a_{0}$ neighbours on $\beta$ in the Voronoi diagram Vor ${ }_{G}$, where $a_{0}$ is the number of aspects of $G_{0}$.

Proof. Let $\pi$ be the orthogonal projection to the subspace $\alpha$. By Lemma 1.3, if $Q \in \beta$ is a neighbour of $P \in \alpha$, then $\operatorname{Vor}_{G_{0} P}(P)$ intersects $\operatorname{Vor}_{G_{0} Q^{\prime}}\left(Q^{\prime}\right)$, where $Q^{\prime}=\pi_{\alpha} Q$. For each aspect of $G_{0}$ there are at most $2^{k}$ points $Q \in \beta$ such that $\operatorname{Vor}_{G_{0}} Q^{\prime}\left(Q^{\prime}\right)$ intersects $\operatorname{Vor}_{G_{0} P}(P)$, by Lemma 1.5 .

Although we do not need this result, the properties we have mentioned so far lead to a short proof of Delone's fundamental theorem of stereohedra for the particular case of Dirichlet stereohedra:

Theorem 1.8 (Delone). Let $G$ be a crystallographic group in $\mathbb{R}^{d}$ with a aspects. Then any Dirichlet stereohedron for the group $G$ has at most $2^{d}(a+1)-2$ facets.

Proof. Let $P$ be a point with trivial stabilizer, let $G P$ be its orbit and let $\operatorname{Vor}_{G P}(P)$ be the Dirichlet stereohedron we want to study. Let $G_{T}$ be the translational subgroup of $G$. We decompose $G P=G_{T} P_{1} \cup \cdots \cup G_{T} P_{a}$, with $P_{1}=P$. Then $\operatorname{Vor}_{G P}(P) \subset \operatorname{Vor}_{G_{T} P}(P)$ and $\operatorname{Vor}_{G_{T} P}(P)$ intersects at most $2^{d}$ regions in each $\operatorname{Vor}_{G_{T} P_{i}}\left(P_{i}\right)$ for $i \neq 1$, which implies that $P$ has at most $2^{d}(a-1)$ neighbours contained in $G P \backslash G_{T} P$.

Let $G_{T}^{2}$ be the subgroup of $G_{T}$ consisting of translations of $G_{T}$ doubled, as in Lemma 1.4. No neighbour of $P$ can be in $G_{T}^{2} P$ and each neighbour in $G_{T} P \backslash G_{T}^{2} P$ will be in the relative interior of a facet of $\operatorname{Vor}_{G_{T}^{2}}(P)$, by arguments similar to the ones in the proof of Lemma 1.4. Since $G_{T}^{2}$ has index $2^{d}$ in $G_{T}$, the "density" of points of $G_{T} P \backslash G_{T}^{2} P$ in each region of $\operatorname{Vor}_{G_{T}^{2}}$ is $2^{d}-1$ points per region. We are interested in the points of $G_{T} P \backslash G_{T}^{2} P$ which lie in the boundary of only two regions of $\operatorname{Vor}_{G_{T}^{2}}$ and the number of those is at most $2\left(2^{d}-1\right)$, as desired.

\section{Dirichlet Stereohedra for Groups with Reflections}

Let $G$ be a crystallographic group with reflections in dimension $d$, let $G_{r}$ be the subgroup of $G$ generated by its reflections. Note that $G_{r}$ is not necessarily crystallographic, since it might not contain $d$ linearly independent translations.

Definition 2.1. A reflection cell of $G$ is the minimal region of $\mathbb{R}^{d}$ with all facets lying in reflection hyperplanes. Equivalently, a reflection cell is a connected component of the complement of the union of all the reflection hyperplanes of $G$.

The reflection cells are all congruent to one another and tile the space, since they are Dirichlet domains of $G_{r}$. The reflection cells are bounded if and only if $G$ has reflections in $d$ independent directions. Equivalently, if $G_{r}$ is crystallographic. No point 


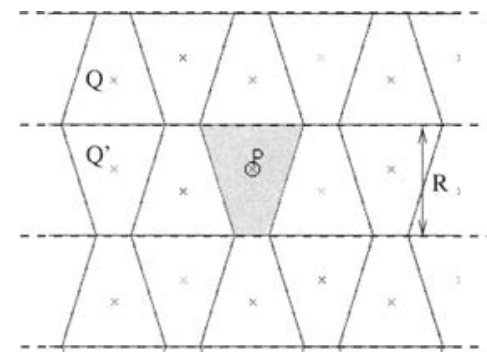

Fig. 1. Proposition 2.2.

in a reflection plane of the group $G$ can lie in an open Voronoi region of $\operatorname{Vor}_{G P}$. Hence, Vor $_{G P}$ is a refinement of the tiling consisting of the reflection cells. Moreover:

Proposition 2.2. Let $R$ be a reflection cell for $G$ and $P \in R$. Let $S=G P \cap R$ be the set of points in the orbit $G P$ of $P$ inside $R$. Then

$$
\operatorname{Vor}_{G P}(P)=R \cap \operatorname{Vor}_{S}(P) \text {. }
$$

Proof. ( $\subseteq$ ) Since $G P \supset S$ we have $\operatorname{Vor}_{G P}(P) \subseteq \operatorname{Vor}_{S}(P)$. In the same way $G P \supset$ $G_{r} P$ and $R=\operatorname{Vor}_{G_{r} P}(P)$ implies $\operatorname{Vor}_{G P}(P) \subseteq R$. Therefore $\operatorname{Vor}_{G P}(P) \subseteq \operatorname{Vor}_{S}(P) \cap R$.

() Let $x \in R \cap \operatorname{Vor}_{S}(P)$ and suppose $Q \in G P \backslash S$ (see Fig. 1). Since there exists a reflection hyperplane $l$ which separates $x$ from $Q$, it follows that there is a point $Q^{\prime} \in G P$ (obtained from $Q$ by reflection in $l$ ) with $d\left(x, Q^{\prime}\right)<d(x, Q)$. If $d_{0}=$ $\min _{Q \in G P}\{d(x, Q)\}$ we have that $d_{0}=\min _{Q \in S}\{d(x, Q)\}=d(x, P)$. Then $d(x, P)<$ $d(x, Q), \forall Q \in G P \Rightarrow x \in \operatorname{Vor}_{G P}(P)$.

Given a point $P$ in a reflection cell $R$ we call those neighbours of $P$ which lie outside (resp. inside) $R$ the external (resp. internal) neighbours of $P$. By Proposition 2.2, the common facet between $\operatorname{Vor}_{G P}(P)$ and the region of an external neighbour is part of a facet of $R$. Therefore $P$ has at most as many external neighbours as the number of facets of $R$. Proposition 2.2 also says that the internal neighbours of $P$ in $\operatorname{Vor}_{G P}(P)$ are exactly the neighbours of $P$ in $\operatorname{Vor}_{G P \cap R}$ whose corresponding region intersects $R$.

Among the 219 affine equivalence classes of three-dimensional crystallographic groups, there are 100 with reflections. We study separately the groups with reflections in three, two and one independent directions. By this we mean, respectively, that the normal vectors to the reflection planes span a three, two or one-dimensional space, respectively.

\subsection{Groups with Reflections in Three Directions}

There are 28 classes of groups with reflections in three directions: 4 in the orthorhombic system, 7 in the tetragonal system, 4 in the hexagonal system, and 13 in the cubic system. Let $G$ be one of them. 
Proposition 2.3. Let $P$ be a point with trivial stabilizer in $G$. Then $P$ has at most six external neighbours and at most five internal neighbours in $V^{\circ} r_{G P}$.

Proof. Every group generated by reflections is a direct product of groups whose fundamental domains are simplexes (see page 190 of [5]). Therefore the reflection cells are products of simplices. The possibilities are a tetrahedron, a triangular prism and a rectangular prism, so that the maximal number of facets of the reflection cell is six. The points of $G P$ which are inside $R$ are all at equal distance to the centroid of $R$, since every isometry $g \in G$ has $g(R)=R$. Hence, the points of $G P \cap R$ lie on a sphere. Their Voronoi diagram induces a monohedral tiling of this sphere and by Euler's formula each region in such a tiling has at most five edges. Hence, the number of internal neighbours of $P$ is at most five.

Theorem 2.4. For any three-dimensional crystallographic group $G$ with reflections in three independent directions and any point $P$ with trivial stabilizer, the Dirichlet stereohedron $\operatorname{Vor}_{G P}(P)$ has at most eight facets.

Proof. Recall from the previous proof that the points of $G P$ inside $R$ lie on a sphere, centred at the centroid $O$ of $R$. More precisely, they form an orbit of a finite discrete group of motions which we call $G_{O}$. Moreover, $G_{O}$ is a subgroup of the symmetry group of the reflection cell $R$ and it does not contain reflections. This implies that the only possibilities for $G_{O}$ are (see pages 124-130 of [14]):

1. A cyclic group consisting of rotations around a line. In this case $P$ has only two internal neighbours.

2. A dihedral group generated by a rotation of order $m(m \in\{1,2,3,4,6\})$ around a line $l$ and either an inversion (if $m$ is odd) or a rotation of order two on a line perpendicular to $l$ (for $m \geq 2$ ). We call the direction of $l$ "vertical." If $m=2, P$ has at most three internal neighbours (since $G_{O}$ has four elements). In any other case $P$ has at most four internal neighbours: two in the horizontal plane containing $P$ and two in another horizontal plane.

For the external neighbours, observe that $R$ has to be a prism over either a square (and $m=2$ or 4 ), a triangle (and $m=3$ ) or a rectangle (and $m=2$ ). $\operatorname{Vor}_{G P}(P)$ is contained in an angular sector of $360 / \mathrm{m}$ degrees around $l$, which implies that it can be incident to at most two vertical facets of $R$ in the cases where $m \neq 2$ and at most three in case $m=2$. This gives at most four external neighbours for $m \neq 2$ and at most five for $m=2$.

3. The group of direct symmetries of either a regular tetrahedron ( 12 elements) or a regular cube ( 24 elements). In both cases $R$ has to be a regular cube, since a regular tetrahedron does not tile the space, and the group $G_{O}$ contains the rotations of order three on the four diagonals $l_{1}, l_{2}, l_{3}$ and $l_{4}$ of the cube. Considering a particular diagonal $l_{i}, \operatorname{Vor}_{G P}(P)$ has to be contained in an angular sector of $120^{\circ}$ at that diagonal. Such a sector intersects at most four facets of the cube (see Fig. 2). More precisely, for each diagonal $l_{i}$ of the cube there are two facets of the cube sharing an edge which does not meet $l_{i}$ and which do not produce facets of $\operatorname{Vor}_{G P}(P)$. This implies that at most three of the facets of the cube produce facets of $\operatorname{Vor}_{G P}(P)$. Hence, $P$ has at most three external neighbours. 


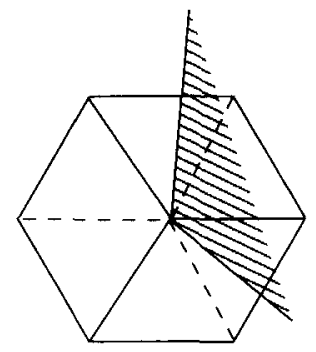

Fig. 2. An angular sector of $120^{\circ}$ at a diagonal of a cube.

Example 2.5. A Dirichlet stereohedron with eight facets, for a group with three independent reflections.

Consider the group $I \frac{4}{m} \frac{2}{c} \frac{2}{m}$ generated by the reflections on the six facets of a square prism and the eight direct symmetries of the prism. Its reflection cell is the square prism and it has eight points of each generic orbit inside the reflection cell, forming the vertices of a square antiprism with bases parallel to those of the prism. In Fig. 3(a) the reflection cell is projected to a square and the vertices of the two square bases of the antiprism are drawn as black and white points respectively. Point $P$ is labelled 1 and the others $2, \ldots, 8$. We call the direction of the prism vertical and the plane containing $P$ upper. Suppose first that the reflection cell is very long in height. Then $P$ has seven neighbours: the four vertices adjacent to it in the skeleton of the antiprism (two in each square, labelled 2,3, 7 and 8 in the figure), its reflection on the top facet of the reflection cell and its reflections on the two vertical facets of the reflection cell closer to $P$. See Fig. 3(b).

Let $O$ be the centre of both the reflection cell and the antiprism with vertices in the orbit. Let $v$ (resp. $v^{\prime}$ and $v^{\prime \prime}$ ) be the endpoint of the edge 128 (resp. 123 and 178) in the boundary of the reflection cell. Clearly, the edges 123, 128 and 178 are all incident to $O$. This implies that $v$ is the lowest point in the region of $P$. If we decrease the height of the reflection cell by moving its two square bases simultaneously towards the centre, the first combinatorial change in the Voronoi region of $P$ will occur when the bottom reflection plane cuts off the vertex $v$ and the top reflection plane traverses the vertices $v^{\prime}$

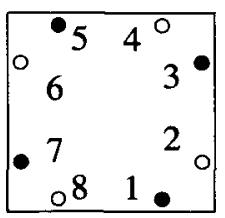

(a)

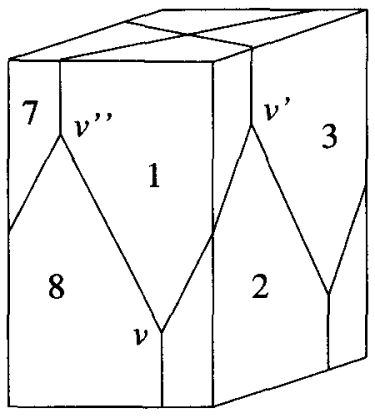

(b)

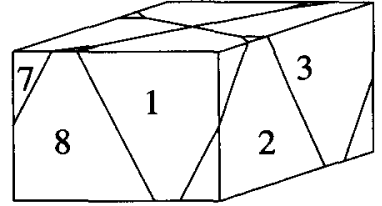

(c)

Fig. 3. A Dirichlet stereohedron with eight facets for a group with three independent reflections. 
and $v^{\prime \prime}$. The change in $v^{\prime}$ and $v^{\prime \prime}$ does not affect the number of neighbours of $P$, but the change in $v$ increases the number by one, since the bottom reflection plane now creates a facet in the Voronoi region. This is shown in Fig. 3(c), where region 1 is still a neighbour of regions 2, 3, 7 and 8 and it has four facets on the boundary of the prism.

\subsection{Groups with Reflections in Two Directions}

There are 40 groups with reflections in two directions: 10 in the orthorhombic system, 19 in the tetragonal system, 6 in the trigonal system and 5 in the hexagonal system. Let $G$ be one of them and let $R$ be one of its reflection cells. Let $\alpha$ be a plane perpendicular to all the reflection planes. The projection of $R$ on the plane $\alpha$ is a bounded reflection cell of a two-dimensional crystallographic group, i.e. one of the five triangles or quadrilaterals shown in Fig. 4. $R$ is an infinite prism having as "base" one of the five polygons.

The points inside the prism $R$ form a finite family of one-dimensional lattices in the direction orthogonal to $\alpha$, and the number of these lattices cannot exceed the number of symmetries of the planar projected reflection cell, since every element of $G$ sending $P$ to a point inside $R$ fixes $R$. The maximum number of possible one-dimensional lattices is shown by black dots in each case in Fig. 4 . The extremal case is that of a square, in which there are eight such dots.

Proposition 2.6. Given a group $G$ with reflections in two independent directions, any point $P$ with trivial stabilizer has at most most 16 internal neighbours and at most four external neighbours in $\operatorname{Vor}_{G P}$.

Proof. Since $R$ has at most four facets, $P$ has at most four external neighbours. By Proposition 1.7, $P$ has at most two internal neighbours in each of the one-dimensional lattices of $G P$ inside $R$, hence it has at most 16 internal neighbours.

Theorem 2.7. For any three-dimensional crystallographic group $G$ with reflections in two independent directions and any point $P$ with trivial stabilizer, the Dirichlet stereohedron $\operatorname{Vor}_{G P}(P)$ has at most 18 facets.

Proof. We only need to deal with the case where the number of one-dimensional lattices inside $R$ is exactly eight, i.e. the case represented on the rightmost picture of Fig. 4 . We will prove that one of the following two things occurs: either $P$ has no neighbour in the one-dimensional lattice containing it (in which case it has at most 16 internal neighbours) or $P$ has at most two external neighbours.
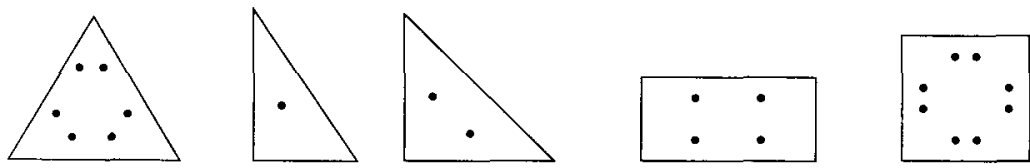

Fig. 4. Bounded planar reflection cells. 


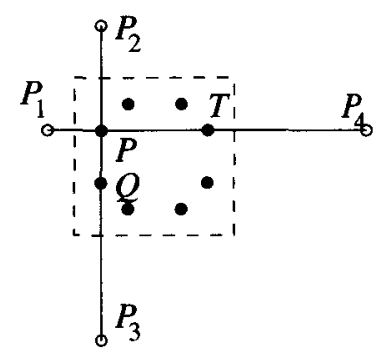

Fig. 5. $P$ has at most 16 neighbours.

To prove this, suppose that $P$ is a neighbour of one of the points in its one-dimensional lattice. Hence, by Proposition 1.2 it is a neighbour of two points in that lattice. If we look at the Delaunay triangulation of $G P$, this implies that the line orthogonal to $\alpha$ and containing $P$ consists of Delaunay edges. By the action of the symmetry group, the same occurs with all the other lines orthogonal to $\alpha$ containing points of $G P$. The end of the proof is illustrated in Fig. 5. The points $P_{3}$ and $P_{4}$, reflected images of $P$ by the two facets of $R$ further away from $P$, cannot be neighbours of $P$ since this would produce Delaunay edges crossing two of the lines orthogonal to $\alpha$.

For our next example we need the following result:

Lemma 2.8. Let $g$ be the screw rotation of order 8 around the vertical axis $x=y=0$, with translation of length 1 , i.e. $g(x, y, z)=((x-y) / \sqrt{2},(x+y) / \sqrt{2}, z+1)$. Let $\langle g\rangle$ be the (infinite cyclic) group generated by $g$. Then any point $P$ not in the axis of $g$ has 16 neighbours in $\operatorname{Vor}_{\langle g\rangle}(P)$.

Proof. Without loss of generality we can assume $P=(a, 0,0)$, with $a>0$.

We denote $P_{i}=g^{i}(P)$ for every $i \in \mathrm{Z}$. We will prove that $P\left(=P_{0}\right)$ is a neighbour of the 16 points $P_{i}$ with $i \in\{-8, \ldots, 8\}, i \neq 0$. It is clear that $P$ is always a neighbour of $P_{1}$ since $P$ and $P_{1}$ are equidistant to $\left(0,0, \frac{1}{2}\right)$ and closer to it than any other $P_{i}$. The same argument works for $P_{-1}$.

Consider now the following quadruples of points: $\left\{P_{-2}, P_{-1}, P_{1}, P_{2}\right\},\left\{P_{-3}, P_{-2}\right.$, $\left.P_{2}, P_{3}\right\},\left\{P_{-4}, P_{-3}, P_{3}, P_{4}\right\}$. By symmetry, the centres of spheres passing through them lie all in the $x$-axis. Their coordinates are

$$
O_{1}=\left(\frac{-3}{\sqrt{2} a}, 0,0\right), \quad O_{2}=\left(\frac{-5}{\sqrt{2} a}, 0,0\right) \quad \text { and } \quad O_{3}=\left(\frac{-7}{(2-\sqrt{2}) a}, 0,0\right)
$$

For each quadruple, we check that every $P_{i}$ not in the quadruple is further from the sphere centre than the ones in the quadruple. We only need to check this for the points $P_{0}=(a, 0,0), P_{1}=(a / \sqrt{2}, a / \sqrt{2}, 1), P_{2}=(0, a, 2), P_{3}=(-a / \sqrt{2}, a / \sqrt{2}, 3)$ and $P_{4}=(-a, 0,4)$. The ones with $i \in\{-4,-3,-2,-1\}$ are obtained by a rotation around the line $y=z=0$ in which the three centres lie, and the rest of the $P_{i}$ 's are clearly further from that line. We have

$$
d\left(P_{0}, O_{1}\right)^{2}-d\left(P_{1}, O_{1}\right)^{2}=3 \sqrt{2}-4
$$




$$
\begin{aligned}
d\left(P_{1}, O_{1}\right)^{2} & =d\left(P_{2}, O_{1}\right)^{2} \\
d\left(P_{3}, O_{1}\right)^{2}-d\left(P_{1}, O_{1}\right)^{2} & =2 \\
d\left(P_{4}, O_{1}\right)^{2}-d\left(P_{1}, O_{1}\right)^{2} & =12-3 \sqrt{2} \\
d\left(P_{0}, O_{2}\right)^{2}-d\left(P_{2}, O_{2}\right)^{2} & =5 \sqrt{2}-4 \\
d\left(P_{1}, O_{2}\right)^{2}-d\left(P_{2}, O_{2}\right)^{2} & =2 \\
d\left(P_{2}, O_{2}\right)^{2} & =d\left(P_{3}, O_{2}\right)^{2} \\
d\left(P_{4}, O_{2}\right)^{2}-d\left(P_{2}, O_{2}\right)^{2} & =12-5 \sqrt{2} \\
d\left(P_{0}, O_{3}\right)^{2}-d\left(P_{3}, O_{3}\right)^{2} & =\frac{16 \sqrt{2}-4}{2-\sqrt{2}} \\
d\left(P_{1}, O_{3}\right)^{2}-d\left(P_{3}, O_{3}\right)^{2} & =\frac{22 \sqrt{2}-16}{2-\sqrt{2}} \\
d\left(P_{2}, O_{3}\right)^{2}-d\left(P_{3}, O_{3}\right)^{2} & =\frac{12 \sqrt{2}-10}{2-\sqrt{2}}, \\
d\left(P_{3}, O_{3}\right)^{2} & =d\left(P_{4}, O_{3}\right)^{2} .
\end{aligned}
$$

This implies that each pair of points in the same quadruple are neighbours in $\operatorname{Vor}_{G P}(P)$. By symmetry, if $P_{i}$ and $P_{j}$ are neighbours, then $P_{j-i}$ is a neighbour of $P_{0}$. This finishes the proof.

Example 2.9. A Dirichlet stereohedron with 18 facets, for a group with two independent reflections.

Let $G$ be a group of type $I \frac{4_{1}}{g} \frac{2}{m} \frac{2}{d}$ whose reflection cell is an infinite orthogonal prism over a square and generated by the following isometries:

1. Reflections on the four planes containing facets of the infinite prism. Assume without loss of generality that these planes are given by the equations $x=k$, $x=-k, y=k$ and $y=-k$ for a certain $k>0$.

2. A screw rotation $\rho$ of order 4 around the axis of the prism. We assume without loss of generality that $\rho$ sends $(x, y, z) \mapsto(-y, x, z+2)$.

3. A rotation $\sigma$ of order 2 on a horizontal axis and which fixes the prism. Without loss of generality we take this axis to be $y=z=0$, so that the rotation is $(x, y, z) \mapsto(x,-y,-z)$.

For any base point $P$ in the reflection cell, the points in $G P \cap R$ are the point $Q=\sigma P$ obtained by the order-2 rotation and the points $\rho^{i} P$ and $\rho^{i} Q(i \in \mathrm{Z})$ obtained by the screw rotation. We take as base point $P=\left(a \cos (\pi / 8), a \sin (\pi / 8), \frac{1}{2}\right)$ for a certain $a>0$. Then $\sigma(P)=\left(a \cos (-\pi / 8), a \sin (-\pi / 8),-\frac{1}{2}\right)$, which is the same point obtained by applying the inverse of the screw rotation $g$ of Lemma 2.8 to $P$. This implies that $G P \cap R$ is exactly the orbit $\langle g\rangle P$ which appears in Lemma 2.8 (we are using that $g^{2}=\rho$ ). The lemma implies that taking $k$ sufficiently large, the Dirichlet region of $P$ will have 16 facets in the interior of the prism. In order to obtain a concrete example, it is sufficient that $k$ be such that the three sphere centres $O_{1}, O_{2}$ and $O_{3}$ in the proof of 
Lemma 2.8 are inside the reflection cell. That is, it is sufficient that

$$
k>\frac{7}{(2-\sqrt{2}) a} \simeq \frac{11.95}{a} .
$$

Moreover, we can easily prove that the Dirichlet region has at least two external facets: Since all the Dirichlet regions inside the prism are equivalent, all of them have the same number of external facets. If all of them have only one external facet, then there have to be some vertical facets in the Voronoi diagram inside $R$. However, this would imply that there are points in $G P \cap R$ with the same height, which is not the case: there is exactly one point in $G P \cap R \cap\left\{z=m+\frac{1}{2}\right\}$ for each integer $m \in Z$.

\subsection{Groups with Reflections in One Direction}

There are 32 classes of three-dimensional crystallographic groups with reflections in one direction: 5 in the monoclinic system, 16 in the orthorhombic system, 6 in the tetragonal system and 5 in the hexagonal system. Let $G$ be any one of them, let $R$ be a reflection cell of $G$ and let $P$ be a point in $R$ and with trivial stabilizer. The reflection cell $R$ is an infinite strip determined by two parallel reflection planes which we assume are horizontal and at heights 1 and -1 (see Fig. 6). Therefore the maximal number of external neighbours is at most two.

Let $h$ be the height of the horizontal plane which contains the point $P \in R$. Since every element of $G$ brings horizontal planes to horizontal planes, all the points of the orbit $G P$ lie on planes at distance $1-h$ from some reflection plane. Then, inside $R$ we have points of $G P$ only on the planes $h$ and $-h$. If $h=0, h$ and $-h$ is the same plane (see Fig. 6).

Let $G_{0}=\{g \in G: g(\alpha)=\alpha$, for every horizontal plane $\alpha\}\left(G_{0}\right.$ is the subgroup of $G$ which contains the "horizontal motions"). We study the number of internal neighbours of $P$ separately among points of $G P$ on the planes $h$ and $-h$ (if $h=0$, the second is not necessary).

Lemma 2.10. $P \in h$ has at most six neighbours on the plane $h$.

Proof. Let $G_{h}=\{g \in G: g(h)=h\}$, then $G P \cap h=G_{h}(P)$.

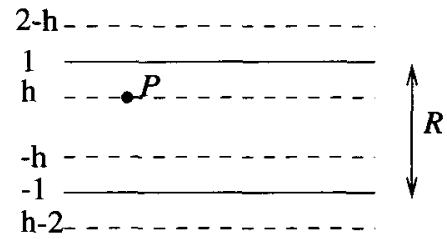

(a)

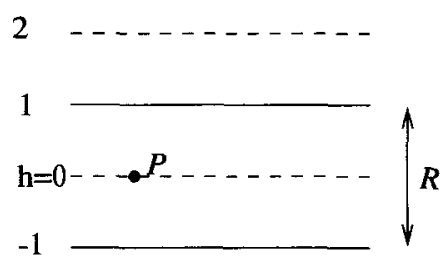

$-2 \ldots \ldots \ldots \ldots$

(b)

Fig. 6. Reflection planes for groups with reflections in one direction. (a) $h \neq 0$, (b) $h=0$. 
If $Q \in h$ is a neighbour of $P \in h$ in the three-dimensional Delaunay triangulation there exists a sphere $\mathcal{S}$ passing through $P$ and $Q$ with no point from the orbit $G P$ of $P$ in its interior. The circle $\mathcal{S} \cap h$ in the plane $h$ passes through $P$ and $Q$ and has no interior point from $G P \cap h$, therefore $Q$ is a neighbour of $P$ also in the two-dimensional Dirichlet tessellation of $G_{0} P$ in the plane $h$. The maximum number of neighbours of a point in a two-dimensional stereohedral tessellation is six.

Lemma 2.11. $P \in h$ has at most seven neighbours on the plane $-h$.

Proof. Suppose $h \neq 0$; otherwise the statement follows from Lemma 2.10 .

Observe that $G P \cap h=G_{0} P$ and, for any $Q \in G P \cap(-h), G P \cap(-h)=G_{0} Q$. By Lemma 1.3, we can bound the number of neighbours of $P$ in the plane $-h$ by the number of regions of $\operatorname{Vor}_{G_{0} P}(Q)$ intersected by $\operatorname{Vor}_{G_{0} P}(P)$, either considered as threedimensional Voronoi diagrams or as two-dimensional Voronoi diagrams on the same plane. Moreover, $G_{0} P \cup G_{0} Q$, when projected to the horizontal plane at height 0 , forms an orbit of the subgroup of $G$ which fixes this plane. By Theorem 3.1 of the next section, $\operatorname{Vor}_{G_{0} P}(P)$ intersects at most seven regions of $\operatorname{Vor}_{G 0 P}(Q)$.

Adding up the bounds for internal and external neighbours we conclude that:

Theorem 2.12. For any three-dimensional crystallographic group $G$ with reflections in only one independent direction and for any point $P$ with trivial stabilizer, the Dirichlet stereohedron $\operatorname{Vor}_{G P}(P)$ has at most 15 facets.

Example 2.13. A Dirichlet stereohedron with 15 facets, for a group with only one independent reflection.

Let $G_{0}$ be a two-dimensional crystallographic group of type $p 2$ generated by rotations of order 2 on the four vertices $(0,0),(0,1),(1,0)$ and $(1,1)$ of a square. The shaded region in Fig. 7 is the Dirichlet region of the point $\left(\frac{1}{2}, \varepsilon\right)$ for a certain $\varepsilon \in\left(\frac{1}{2}, 1\right)$, whose orbit is represented by black dots. The Voronoi diagram of the orbit of $\left(\varepsilon, \frac{1}{2}\right)$ (white dots) is also shown. The small ovals in the figure represent rotation centres of order 2 . The Dirichlet region of one tessellation intersects seven regions of the other, and the two orbits together form an orbit of a group $\mathrm{cmm}$.

Now let $G$ be a three-dimensional crystallographic group of type $P \frac{2}{m} \frac{2}{c} \frac{2}{c}$ having $G_{0}$ as its subgroup of horizontal translations and generated by $G_{0}$ together with

- the reflections in parallel horizontal planes $z=k$ and $z=-k$ and

- the rotation of order 2 on the axis $x=y=0$, which fixes one of the reflection cells exchanging its two boundary planes.

Let $P=\left(\frac{1}{2}, \varepsilon, h\right)$ and $Q=\left(\varepsilon, \frac{1}{2},-h\right)$. Figure 7 can be regarded as the projection of the two orbits $G_{0} P$ and $G_{0} Q$ considered in the proof of Lemma 2.11. If the two reflection planes bounding $R$ are sufficiently far, then $P$ has exactly six neighbours on the plane $h$, seven on the plane $-h$ and one facet in the top reflection plane. If we move closer to the two reflection planes, the first combinatorial change occurs when the bottom reflection 


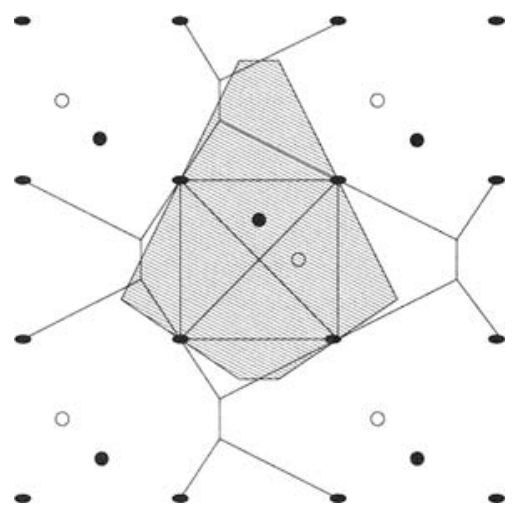

Fig. 7. The Dirichlet intersection number of a group $p 2$ is at least seven.

plane crosses the lowest vertex of $\operatorname{Vor}_{G P}(P)$, which increases by one the number of neighbours, in a way similar to Example 2.5.

\section{How Two Two-Dimensional Dirichlet Tessellations of the Same Group Intersect}

The proof of Lemma 2.11 relied on the answer to the following question: Given two different orbits $G P$ and $G Q$ of the same two-dimensional crystallographic group $G$, how many regions of the Dirichlet tessellation $\operatorname{Vor}_{G Q}$ can each region of $V r_{G P}$ intersect? We call this number the Dirichlet intersection number of the group $G$.

In this section we prove that the Dirichlet intersection number of every planar crystallographic group is bounded above by seven, except perhaps for groups of type pgg. For $p g g$ we have to make the extra assumption that the union $G P \cup G Q$ is again an orbit of a crystallographic group, which is the case that we needed in Lemma 2.11. The following statement summarizes our results:

Theorem 3.1. Let $G$ be a planar crystallographic group and let $G P$ and $G Q$ be two orbits of points with trivial stabilizer. Then the number of Dirichlet regions of one of the orbits intersected by each Dirichlet region of the other orbit is bounded above by:

- one, for groups of types pmm, p3m $1, p 4 m$ and $p 6 m$,

- two, for groups of types cm, cmm, p31m and p4g,

- four, for groups of types $p 1, p 3, p 4, p 6, p m g$ and pm,

- seven, for the groups $p 2$ and $p g$, and

- seven, for the group pgg, if $G P \cup G Q$ is again an orbit of a two-dimensional crystallographic group.

The bounds are tight in all cases except perhaps for pg and pgg.

Proof. The groups of types $p 2, p 3, p 4, p 6, p g$ and $p g g$ require an elaborate proof, and are dealt with in Sections 3.1-3.4. For the other 11 classes of planar crystallographic 
groups, we have:

- Lemma 1.5 directly implies that the number is at most four for any group $p 1$, with one aspect. Independently of the metric parameters of the group, if the base points $P$ and $Q$ for the two orbits are chosen sufficiently generic, then the Voronoi region $\operatorname{Vor}_{G P}(P)$ will intersect exactly four regions of $\operatorname{Vor}_{G Q}$.

- Proposition 2.2 implies that the Dirichlet tessellation for a group generated by reflections does not depend on the chosen orbit, so the Dirichlet intersection number for the groups $p 6 m, p 4 m, p 3 m 1$ and $p m m$ equals one.

- For the other groups which contain reflections in two independent directions $(\mathrm{p} 31 \mathrm{~m}$, $p 4 \mathrm{~g}$ and $\mathrm{cmm}$ ), all the points of the orbit inside a reflection cell $R$ must have the same distance to the centroid of $R$ (every motion sending $R$ to itself fixes the centroid). Hence, $R \cap G P$ and $R \cap G Q$ are orbits of the same finite discrete group and, clearly, a region in one Voronoi diagram intersects two regions in the other, except in degenerate cases where the two Dirichlet tessellations coincide.

- For the three groups which contain reflections in only one direction $(\mathrm{cm}, \mathrm{pm}$ and $p m g$ ), the part of $G P$ (or of $G Q$ ) inside $R$ consists of one (for $\mathrm{cm}$ ) or two (for $p m$ and $p m g$ ) one-dimensional translational lattices, placed in lines parallel to the reflection lines. Using Lemmas 1.3 and 1.5 we conclude that each region of Vor $_{G P}$ can intersect only the regions of at most two points in each one-dimensional lattice. Hence the Dirichlet intersection number is at most two for $\mathrm{cm}$ and at most four for $p m$ and $p m g$. It is easy to check that both bounds are tight.

Observe that the groups $p g$ and $p 2$ have only two aspects, so Corollary 1.6 already implies that their Dirichlet intersection number is at most eight.

Remark 3.2. We conjecture that the Dirichlet intersection number of $p g g$ is at most seven, too. In Section 3.3 we prove it to be at most eleven. Applying the methods used there in a more sophisticated way we can show that it is at most nine, but we do not include a proof of this.

The number seven equals the maximum number of facets of a two-dimensional Dirichlet stereohedron plus one. It is an interesting possibility that the Dirichlet intersection number of any crystallographic group in any dimension might be bounded above by the maximum number of facets of a Dirichlet stereohedron for that group plus one.

\subsection{Groups of Type $p 3, p 4$ and $p 6$}

Proposition 3.3. If $G \in\{p 3, p 4, p 6\}$ and $G P, G Q$ are orbits of $G$, then $\operatorname{Vor}_{G P}(P)$ intersects at most four regions of $\operatorname{Vor}_{G Q}$.

Proof. For any of these three groups, the action of $G$ tiles $\mathbb{R}^{2}$ into two classes of triangles with vertices in rotation centres of the group, as shown in Fig. 8. We call them "black" and "white," respectively. Any black triangle is adjacent to three white ones and vice versa. The union of any pair of adjacent black-white triangles is a fundamental domain for the group. 


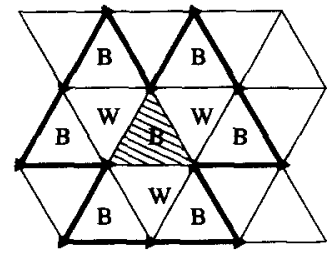

p3

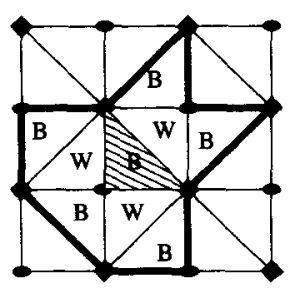

$p 4$

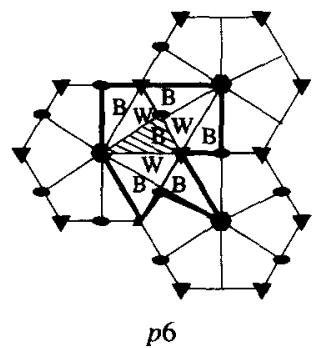

$p 6$

Fig. 8. The groups $p 3, p 4$ and $p 6$ tile the plane into "black" and "white" triangles.

Without loss of generality we suppose $P$ in a black triangle $C_{1} C_{2} C_{3}$. Considering the bisectors between $P$ and the points of $G P$ obtained from $P$ by the rotations at $C_{1}, C_{2}$ and $C_{3}$ we conclude that the Voronoi region of $P$ is contained in the union of $C_{1} C_{2} C_{3}$ and its three adjacent white triangles. If $Q$ is in a white triangle, this argument shows that $\operatorname{Vor}_{G P}(P)$ intersects only the three regions of $\operatorname{Vor}_{G Q}$ corresponding to points in the triangles adjacent to $C_{1} C_{2} C_{3}$. Hence, in what follows we assume without loss of generality that $P$ and $Q$ lie both in the same black triangle $C_{1} C_{2} C_{3}$.

In this case, our previous argument implies that $\operatorname{Vor}_{G P}(P)$ can intersect only the following regions in $\operatorname{Vor}_{G Q}$ : the region of $Q$ and the five (in $p_{4}$ and $p_{6}$ ) or six (in $p_{3}$ ) regions corresponding to points of $G Q$ in the black triangles at distance two from $C_{1} C_{2} C_{3}$. These are the triangles contained in the thick polygons of Fig. 8. We prove now that only three from the six or five black triangles other than $C_{1} C_{2} C_{3}$ produce regions which intersect $\operatorname{Vor}_{G P}(P)$, for each fixed $P$ and $Q$. For this observe that the structure of the Dirichlet tessellation $\operatorname{Vor}_{G P}$ for any point $P$ in the interior of $C_{1} C_{2} C_{3}$ is as follows: each region consists of a full black triangle and part of its three adjacent white triangles, in such a way that a unique new vertex of the tessellation, apart from the rotation centres, appears in the interior of any white triangle. See Fig. 9, where this is shown for the group $p 4$.

Of course, the decompositions of the white triangles into three subtriangles are all equivalent by the action of the group. In a generic situation (and we only need to consider
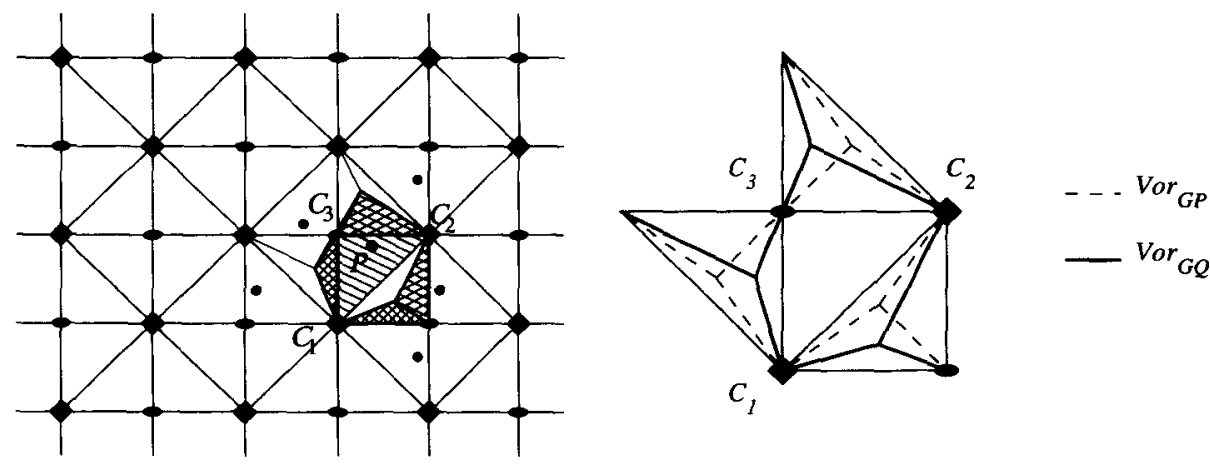

Fig. 9. The Dirichlet intersection number of $p 4$ is four. 
this case since degeneracies can only decrease the number of regions intersected) exactly one of the new vertices of $\operatorname{Vor}_{G P}$ will be contained in $\operatorname{Vor}_{G Q}(Q)$. Say that it is the vertex in the white triangle incident to the edge $C_{1} C_{2}$, as in Fig. 9. Then $\operatorname{Vor}_{G} P(P)$ does not intersect any Voronoi region of $\operatorname{Vor}_{G Q}$ other than $\operatorname{Vor}_{G Q}(Q)$ in that white triangle. With similar arguments, in the other two white triangles $\operatorname{Vor}_{G P}(P)$ intersects respectively one and two regions of $\operatorname{Vor}_{G Q}$ other than $\operatorname{Vor}_{G Q}(Q)$.

Actually, using the conventions of "black" and "white" triangles the proof of the previous lemma says:

Corollary 3.4. If $G \in\{p 3, p 4, p 6\}$ and $G P, G Q$ are orbits of $G$, such that $Q$ and $P$ do not belong to a triangle of the same colour, then $\operatorname{Vor}_{G P}(P)$ intersects three regions of $\operatorname{Vor}_{G Q}$. If they belong to triangles of the same colour and they are sufficiently generic, then $\operatorname{Vor}_{G P}(P)$ intersects four regions of $\operatorname{Vor}_{G Q}$.

\subsection{Groups of Type $p 2$}

Let $G$ be a group of type $p 2$, i.e. generated by two translations of vectors which we denote $a$ and $b$ and a rotation of order 2 . The order- 2 rotation centres of $G$ form an affine twodimensional lattice and the translation vectors are exactly twice the differences between rotation centres. The plane can be naturally tiled with congruent triangles with vertices in the rotation centres and without obtuse angles. There is a unique way of doing this, unless the translation vectors $a$ and $b$ are orthogonal, in which case we choose one of the two possibilities. Any of these triangles, together with its three adjacent ones, forms a fundamental domain for $G$. One is shown in Fig. 10.

We divide each of the triangles into three "ortho-regions" labelled $A, B$ and $C$ by drawing the altitudes from the vertices to the orthocentre (see again Fig. 10). For symmetry reasons we rather speak of rhombi of types $A, B$ or $C$, where a rhombus is the union of two adjacent ortho-regions labelled with the same letter.

For any choice of the point $P$ in a certain rhombus labelled $A$, the region $\operatorname{Vor}_{G P}(P)$ is contained in the shaded region of Fig. 11(a). We call this the extended Dirichlet region associated to the rhombus $A$. To prove this assertion, the rest of the plane has been is divided into pieces associated to certain points in the orbit $G P$, displayed in the figure. It is easy to check that the piece containing a certain point $P^{\prime} \in G P$ consists of points

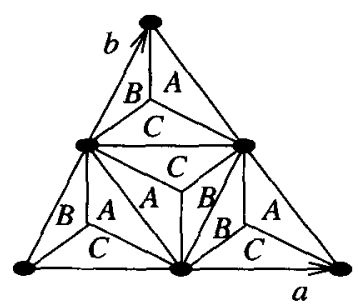

Fig. 10. A fundamental domain for $G=p 2$. 


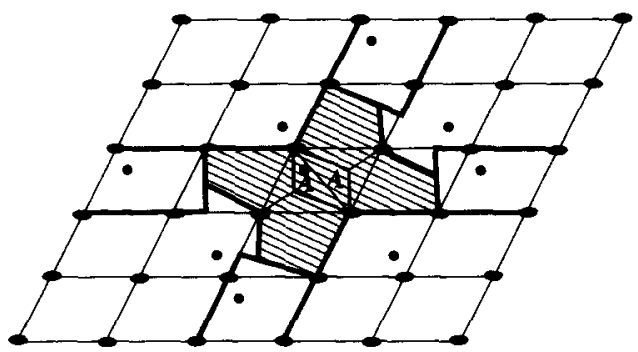

(a)

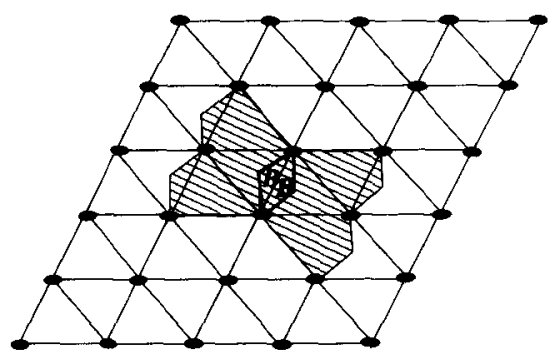

(b)

Fig. 11. Extended Dirichlet regions for the rhombi $A$ and $B$.

which are closer to $P$ than to $P^{\prime}$ for any choice of $P$ in the rhombus. The way to verify this is to consider the bisector of $P$ and $P^{\prime}$, for varying $P$ (taking into account in each case the isometry sending $P$ to $P^{\prime}$ ). Part (b) of the same figure shows the extended Dirichlet region of a rhombus labelled $B$.

Of course, we can consider the extended Dirichlet region for any other rhombus of type $A, B$ or $C$. In order to bound the number of regions of $V o r_{G Q}$ intersected by $\operatorname{Vor}_{G P}(P)$ we use the obvious fact that if $\operatorname{Vor}_{G P}(P) \cap \operatorname{Vor}_{G Q}\left(Q^{\prime}\right) \neq \emptyset$, then the extended Dirichlet regions of the rhombi containing $P$ and $Q^{\prime}$ overlap. Without loss of generality we suppose that $P$ lies in a rhombus labelled $A$ and we consider the cases of $Q$ lying in a rhombus $A$ or $B$ separately. The case of a rhombus $C$ would be analogous to $B$.

\section{$[A, A]$}

There are 13 rhombi of type $A$ whose extended Dirichlet region intersects a given one also of type $A$. The easiest way to see that this is true is to observe that the initial extended Dirichlet region is contained in the union of a parallelogram with its vertices in rotation centres and the four parallelograms adjacent to it. Hence, the rhombi we are interested in are contained in the "Aztec diamond" formed by these five parallelograms and the eight ones adjacent to them. This is shown in Fig. 12.

Now, these 13 rhombi belong to two orbits by the action of $G$, one with nine rhombi (shaded in Fig. 12) and the other with only four. Hence, we only need to deal with the case where $G Q$ lies in the first orbit of the rhombi. In this case, without loss of generality we suppose that $P$ and $Q$ lie in the same rhombus, which we denote $A_{P}$.

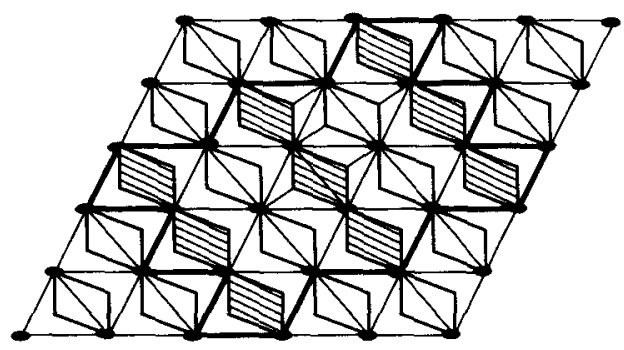

Fig. 12. Possible intersecting extended Dirichlet regions in the case $[A, A]$. 


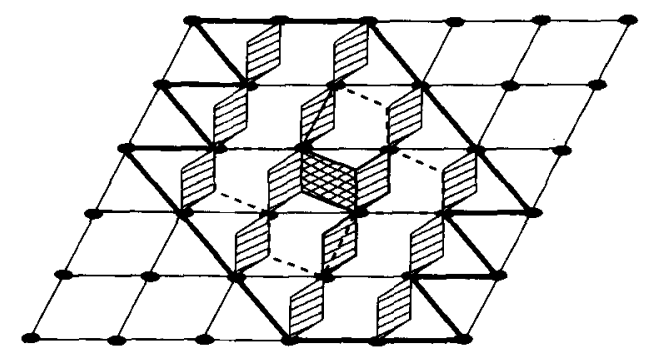

Fig. 13. Possible intersecting extended Dirichlet regions in the case $[A, B]$.

The other eight shadowed rhombi are the ones obtained by the four rotations on the vertices of the parallelogram containing $A_{P}$ and the four translates $\tau_{a}\left(A_{P}\right), \tau_{-a}\left(A_{P}\right)$, $\tau_{b}\left(A_{P}\right)$ and $\tau_{-b}\left(A_{P}\right)$. We claim that only one of $\tau_{a}\left(A_{P}\right)$ and $\tau_{-a}\left(A_{P}\right)$ can contain a point of $G Q$ whose region intersects $\operatorname{Vor}_{G P}(P)$ (and the same for $b$ ). This reduces the number of possible intersecting regions from nine to seven, as desired.

To prove the claim, observe that the bisectors of $P$ and $\tau_{a} P$ and of $P$ and $\tau-a P$ form a strip of width $|a|$ which contains $\operatorname{Vor}_{G P}(P)$. In the same way, the bisectors of $Q$ and $\tau_{a} Q$ and of $Q$ and $\tau-a Q$ forms a strip parallel to and of the same width as the previous one, separating $\operatorname{Vor}_{G Q}\left(\tau_{a} Q\right)$ and $\operatorname{Vor}_{G Q}\left(\tau_{-a} Q\right)$. Hence, only one of these two regions can intersect the strip containing $\operatorname{Vor}_{G P}(P)$.

\section{$[A, B]$}

There are 14 rhombi of type $B$ whose extended Dirichlet region intersects a given one of type $A$, as shown in Fig. 13. Again, they divide into two orbits by the action of $G$, each having seven elements. Hence, only seven rhombi can contain points of the same orbit $G Q$.

\subsection{Groups of Type pgg}

Let $G$ be a group of type $p g g$, i.e. generated by two orthogonal glide reflections $\gamma_{a}$ and $\gamma_{b}$, so that $\gamma_{a}^{2}:=\tau_{a}$ and $\gamma_{b}^{2}:=\tau_{b}$ are two orthogonal translations of vectors $a$ and $b$. $G$ contains rotations of order 2 whose centres form a rectangular grid, and any of the primitive rectangles in this grid (whose sides equal half the translation vectors $a$ and $b$ ) is a fundamental domain. See Fig. 14. The two medial axes of the rectangle are the axes of two glide-reflections generating $G$. We call the four subrectangles in which the two medial axes divide a fundamental domain the fundamental subdomains of $G$.

Let $G P$ and $G Q$ be two orbits of $G$. Without loss of generality we assume that $P$ and $Q$ lie in the same fundamental domain $D_{G}$ and denote the fundamental subdomain containing $P$ by $A$. We call the other three fundamental subdomains in $D_{G}$, in a circular order, $B, C$ and $D$ (see again Fig. 14).

In the same way as we did for $p 2$, we compute an extended Voronoi region for the fundamental subdomain $A$, i.e. a region containing the Voronoi region $\operatorname{Vor}_{G P}(P)$ for every point $P \in A$. The region obtained is shaded in Fig. 15. As in Fig. 11(a), the rest 


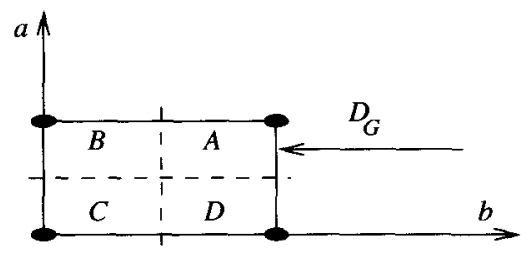

Fig. 14. A fundamental domain for $G=p g g$.

of the plane is divided into pieces each containing a distinguished element of $G P$ so that for any choice of the point $P \in A$ the piece of a point $P^{\prime}$ contains only points closer to $P^{\prime}$ than to $P$.

We can consider the extended Dirichlet region for any other fundamental subdomain in the plane. We call the union of all the subdomains whose extended Dirichlet region intersects the extended Dirichlet region of $A$ the influence region of the subdomain $A$. Clearly, any point $Q^{\prime} \in G Q$ such that $\operatorname{Vor}_{G Q}\left(Q^{\prime}\right) \cap \operatorname{Vor}_{G P}(P) \neq \emptyset$ will lie in the influence region.

To compute the influence region, first observe that it must be contained in the "Aztec diamond" consisting of $D_{G}$, the four fundamental domains adjacent to $D_{G}$, and the eight fundamental domains other than $D_{G}$ adjacent to these four. Then compute the extended Dirichlet region of the 13 shaded subdomains of Fig. 16 (i.e. apply the appropriate transformation of $G$ to the extended Dirichlet region of $A$ ) and check that they do not intersect the extended Dirichlet region of $A$.

The influence region of $A$ contains twelve fundamental subdomains of type $A$, eleven of type $C$ and eight of types $B$ and $D$. Actually, using the same argument as in the proof of the case $[A, A]$ in Section 3.2 the number of subdomains of type $A$ can be reduced to eleven (only two of the four subdomains obtained from $A$ by translation can produce intersecting regions). This immediately shows that the Dirichlet intersection number of $G$ is at most eleven. With more sophisticated arguments we can decrease this to nine, but we do not do it here.

From now on we concentrate in the case when $G P \cup G Q$ is an orbit of a crystallographic group, as needed in Section 2.3.

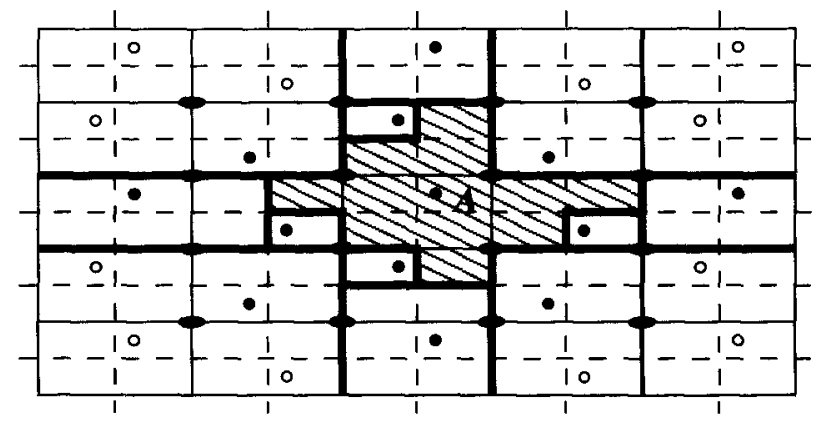

Fig. 15. Extended Dirichlet region for $A$. 


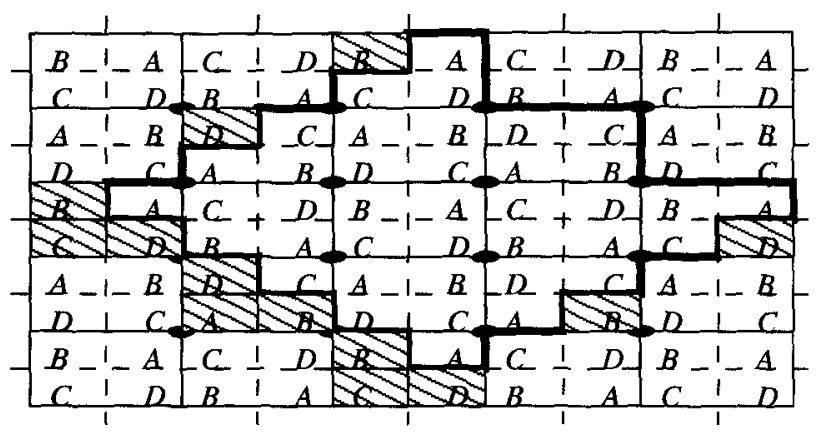

Fig. 16. Influence region of $A$.

Lemma 3.5. Let $G$ be planar crystallographic group of type pgg and let $P$ be a point with trivial stabilizer, in a certain rectangular fundamental domain $D_{G}$. Let $Q \in D_{G}$ be such that the union $G P \cup G Q$ is an orbit of another crystallographic group $H$. Then the element of $H$ sending $P$ to $Q$ is an involutive symmetry of $D_{G}$.

Proof. Let $g$ be the element of $H$ with $g P=Q$. If $P=Q$, then $g$ is the identity and there is nothing to prove. Suppose $P \neq Q$.

Once we prove that $g$ is a symmetry of the rectangle $D_{G}$, the fact that $g$ is an involution is easy: let $g^{\prime} \in H$ such that $g^{\prime} Q=P$. Applying the lemma with $P$ and $Q$ exchanged, we conclude that $g^{\prime}$ is a symmetry of $D_{G}$ too. Now, if $g \neq g^{\prime}$, then $H$ contains two nontrivial elements which fix $D_{G}$ and the index of $G$ in $H$ would be at least three, which is false. Hence, $g=g^{\prime}$ and $g^{2} P=P$. Since $P$ has trivial stabilizer in $G, g^{2}$ is the identity.

We now prove that $g$ is a symmetry of $D_{G}$. We have the following two possibilities: either $H$ has exactly the same rotations of order 2 as $G$, in which case it is clear that $g$ is a symmetry of $D_{G}$ since it preserves the rectangular grid, or $g$ sends the rectangular grid of rotation centres of $G$ to another grid. We assume this is the case. Observe that, then, $H$ is the group generated by $G$ and any of the new rotations.

Any of the two grids of rotation centres is invariant under the rotations of the other one. There are only three ways in which this can occur: (a) the second grid has rotation centres in the centre of the rectangles of the first, (b) the second grid has rotation centres in the midpoints of vertical edges of the rectangles of the first grid and (c) the same with horizontal edges.

In the three cases $H$ contains a non-trivial symmetry of the rectangle $D_{G}$ : In case (a), the rotation of order 2 at the centre of the rectangle $D_{G}$. In case (b), the reflection at the vertical axis of $D_{G}$ and in case (c) the reflection at the horizontal axis. In the last two cases the reflection in question is obtained as a composition of a rotation of order 2 in $H$ and a glide reflection of $G$.

This symmetry of $D_{G}$ sends $P$ to an element of $G Q$ in $D_{G}$, i.e. to $Q$.

There are six possible involutions of $D_{G}$, displayed in Fig. 17: the identity, the rotation of order 2 at the centre of the rectangle, the reflections on the horizontal and vertical axes of the rectangle and, if the rectangle is a square (i.e. if $|a|=|b|$ ) the reflections on 

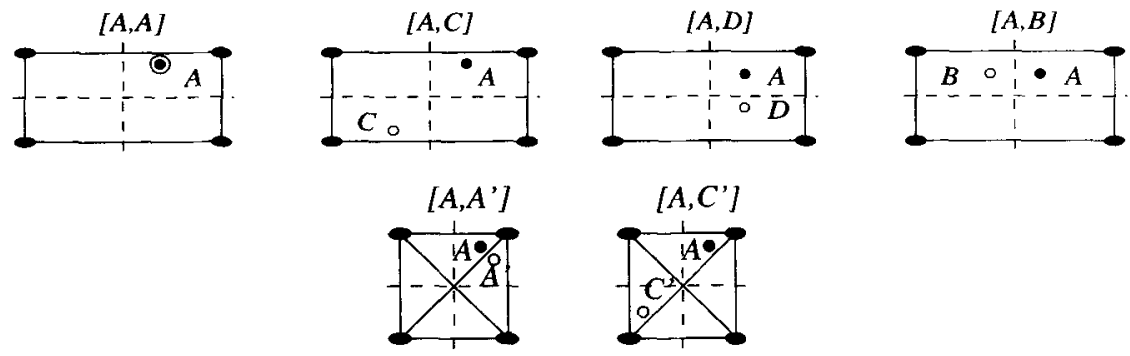

Fig. 17. The six possible involutions of $D_{G}$.

the two diagonals of the square. We name these cases $[A, A],[A, C],[A, B],[A, D]$, $\left[A, A^{\prime}\right]$ and $\left[A, C^{\prime}\right]$, respectively, and study them separately.

\section{$[A, A]$}

$\operatorname{Vor}_{G P}(P)$ intersects only one region of the Voronoi diagram $\operatorname{Vor}_{G Q}$, since $P=Q$.

\section{$[A, B]$ and $[A, D]$}

We just need to discard one of the eight fundamental subdomains of types $B$ and $D$ in the influence region of Fig. 16. We do this with type $B$, since type $D$ is analogous.

Let $P$ be our base point in the subdomain $A$ of our fundamental domain $D_{G}$ and let $Q$ be reflected on the vertical axes, lying in the subdomain of type $B$. Let $P^{\prime}$ and $Q^{\prime}$ denote the points of $G P$ and $G Q$ in the fundamental domain adjacent to the subdomains $B$ and $C$ of $D_{G}$ (the one next to the central one on the left, in Fig. 16). We have $P^{\prime}-P=Q^{\prime}-Q$ so that the bisectors of $P$ and $P^{\prime}$ and of $Q$ and $Q^{\prime}$ are parallel. Moreover, the first one is closer to $P$ than the second one, and hence these two bisectors separate the regions $\operatorname{Vor}_{G P}(P)$ and $\operatorname{Vor}_{G Q}\left(Q^{\prime}\right)$.

\section{$[A, C]$}

In the case $[A, C]$, we want to prove that among the 11 fundamental subdomains of type $C$ inside the influence region there are at most seven whose Voronoi regions intersect $\operatorname{Vor}_{G P}(P)$. In Fig. 18 we show the parts of the orbits $G P$ and $G Q$ which lie inside the influence region, with black and white points, respectively. We will prove that the regions corresponding to the points labelled $2^{\prime}, 4^{\prime}, 5^{\prime}$ and $6^{\prime}$ in Fig. 18 (where $P=0$ and $\left.Q=0^{\prime}\right)$ do not intersect $\operatorname{Vor}_{G P}(P)$.

Observe first that the bisector $b i(0,1)$ is parallel to $b i\left(1^{\prime}, 2^{\prime}\right)$ and with $b i(0,1)$ closer to 0 and $b i\left(1^{\prime}, 2^{\prime}\right)$ closer to $2^{\prime}$. Thus, the Voronoi region of $2^{\prime}$ cannot intersect the one of 0 . In the same way we eliminate the point $4^{\prime}$ using the bisectors $b i(0,3)$ and $b i\left(3^{\prime}, 4^{\prime}\right)$.

In order to show that the Voronoi region of $5^{\prime}$ in $V_{o r} r_{Q}$ does not intersect the one of 0 in $\operatorname{Vor}_{G P}$, we concentrate on the eight points $0,0^{\prime}, 1,1^{\prime}, 3,3^{\prime}, 5$ and $5^{\prime}$, displayed in Fig. 19. The Voronoi diagram of the four points $0,1,3$ and 5 is shown by solid lines. The Voronoi diagram has to be combinatorially as shown in the figure since 0 and 5 are equidistant to the centre of the figure and closer than 3 and 1. The Voronoi diagram of $0^{\prime}, 1^{\prime}, 3^{\prime}$ and $5^{\prime}$ is shown by dashed lines, and obtained from the other one by a reflection in either a vertical or a horizontal line. 


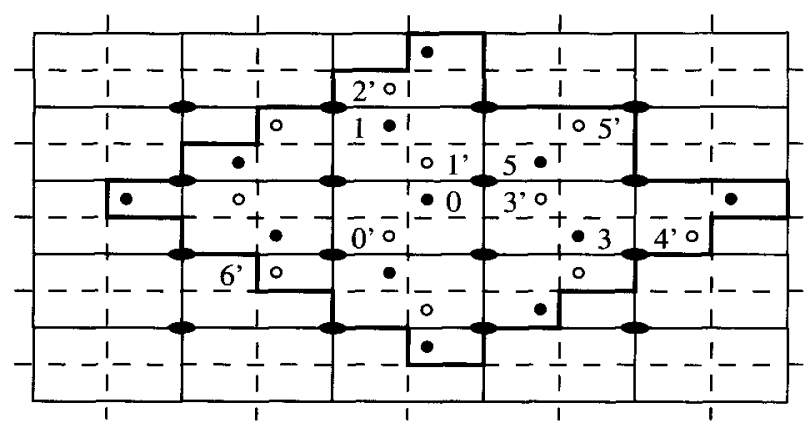

Fig. 18. The case $[A, C]$.

Let $v^{\prime}$ be the unique vertex of the region of $5^{\prime}$ (incident to the regions of $1^{\prime}$ and $3^{\prime}$ ). By symmetry, $v^{\prime}$ is on the same vertical line $r$ as the vertex $v_{r}$ between the regions of 0,3 and 5 and on the same horizontal line $l$ as the vertex $v_{l}$ between the regions of 0,1 and 5 . Then the region $\operatorname{Vor}_{\{0,1,3,5\}}(0)$ is contained in one of the quadrants determined by $l$ and $r$ while $\operatorname{Vor}_{\left\{0^{\prime}, 1^{\prime}, 3^{\prime}, 5^{\prime}\right\}}\left(5^{\prime}\right)$ is contained in the union of the other three quadrants. Hence, the two regions do not intersect, and, by part (ii) of Lemma 1.1, the corresponding regions in $\operatorname{Vor}_{G P}$ and $\operatorname{Vor}_{G Q}$ do not intersect either.

The same reasoning as for $5^{\prime}$, applied taking $6^{\prime}$ as the base point, implies that the regions of 0 and $6^{\prime}$ do not intersect.

\section{$\left[A, A^{\prime}\right]$ and $\left[A, C^{\prime}\right]$}

The remaining cases $\left[A, A^{\prime}\right]$ and $\left[A, C^{\prime}\right]$ only make sense if the fundamental domain is a square, i.e. if the two orthogonal translation vectors $a$ and $b$ of our group have the same length. In this case we compute an extended Dirichlet region again, except that we now divide the fundamental domain into eight congruent triangles instead of four rectangles, as shown in Fig. 20. The extended Dirichlet region for the triangle $A$ is shown on the left, and the associated influence region on the right. There are only six triangles of each of the types $A^{\prime}$ and $C^{\prime}$ inside the influence region of $A$. Hence, the number of regions of $\operatorname{Vor}_{G Q}$ which overlap $\operatorname{Vor}_{G P}(P)$ is at most six.

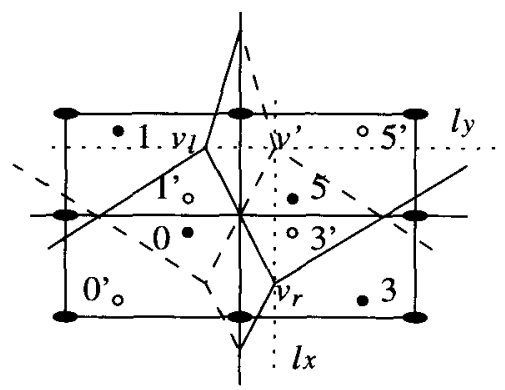

Fig. 19. The case $[A, C] . \operatorname{Vor}_{G Q}\left(5^{\prime}\right)$ does not intersect $\operatorname{Vor}_{G P}(0)$. 


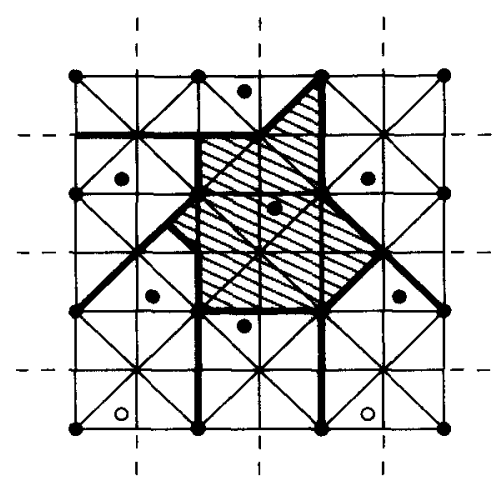

(a)

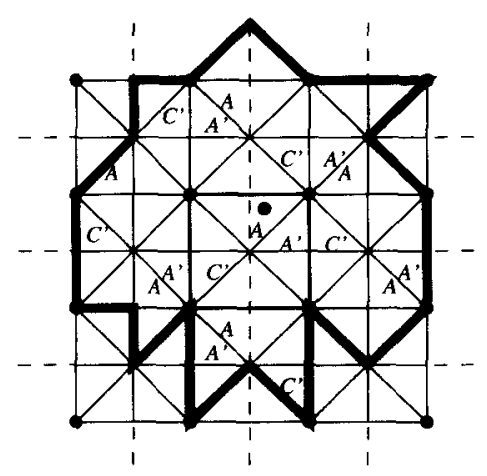

(b)

Fig. 20. For $|a|=|b|$, (a) the extended Dirichlet region for $A$, and (b) the influence region for $A$.

\subsection{Groups of Type pg}

Let $G$ be a planar crystallographic group of type $p g$, i.e. generated by two orthogonal translations of vectors $a$ and $b$ and a family of glide reflections with translation of vector $a / 2$ on lines parallel to $a$ and distant $|b / 2|$ to one another. Any translation of the rectangle $a \times b / 2$ with two sides on glide-reflection axes is a fundamental domain for $G$.

Let $P$ be any point and let $s$ denote the segment parallel to $b$ containing $P$ and joining the closest glide-reflection axes (we assume that $P$ is not on a reflection axis). Using the methods of the previous sections we can easily construct an extended Dirichlet region for the segment $s$, which is shown in Fig. 21(a).

Similarly, we construct the influence region of $s$, defined as the union of all the segments parallel to $b$ and joining two consecutive glide-reflection axes whose extended Dirichlet region intersects the one of $s$. This is shown in Fig. 21(b). The influence region is contained in the union of eight Dirichlet domains of $p g$ : two in between the same glide-reflection axes as $P$ and $s$, two in each of the two adjacent strips between reflection axes and one more in each of the next two strips. In principle this produces a Dirichlet intersection number of eight, but we can reduce it to seven using the same argument as in case $[A, A]$ of group $p 2$ : for any point $Q$, the Voronoi region $\operatorname{Vor}_{G Q}(Q)$ is contained between the bisectors $b i\left(Q, \tau_{b} Q\right)$ and $b i\left(Q, \tau_{b}^{-1} Q\right)$, which form a band of width $|b / 2|$

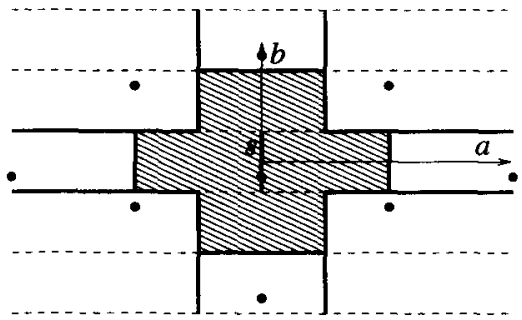

(a)

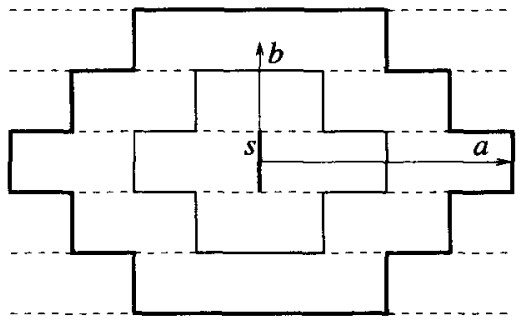

(b)

Fig. 21. Extended Dirichlet region and influence region for the group $p g$. 
parallel to $a$. In particular, only one of the two regions $\operatorname{Vor}_{G Q}\left(\tau_{b} Q\right)$ and $\operatorname{Vor}_{G Q}\left(\tau_{b}^{-1} Q\right)$ can intersect $\operatorname{Vor}_{G P}(P)$. This reduces to one the two possible intersecting regions produced by the two fundamental domains in the strips farther from $s$.

\section{References}

1. D. Bochiş, Estereoedros de Dirichlet en 2 y 3 dimensiones, Ph. D. Thesis, Universidad de Cantabria, 1999.

2. D. Bochiş and F. Santos, On the number of facets of three-dimensional Dirichlet stereohedra, II: non-cubic groups. In preparation.

3. D. Bochiş and F. Santos, On the number of facets of three-dimensional Dirichlet stereohedra, III: cubic groups. Preprint 2000, http://www.matesco.unican.es/ santos/Articulos.

4. G. O. Brunner and F. Laves, How many faces has the largest space-filling polyhedron?, Krist. 147 (1978), 39-43.

5. H. S. M. Coxeter, Regular Polytopes, Dover, New York, 1973.

6. B. N. Delone (or Delaunay), A proof of the fundamental theorem of the theory of stereohedra, Dokl. Akad. Nauk. SSSR 138 (1961), 1270-1272 (in Russian).

7. P. Engel, Geometric Crystallography: An Axiomatic Introduction to Crystallography, Reidel, Dordrecht, 1986.

8. L. Föppl, Der Fundamentalbereich des Diamantgitters, Phys. 15 (1914), 191-193.

9. B. Grünbaum and G. C. Shephard, Tilings with congruent tiles, Bull. Amer. Math. Soc. 3 (1980), 951-973.

10. B. Grünbaum and G. C. Shephard, Tilings and Patterns, Freeman, New York, 1987.

11. D. Hilbert, Mathematische Probleme, in: Gesammelte Abhandlungen, Vol 3, (D. Hilbert, ed.), SpringerVerlag, Berlin, 1935, pp. 290-329.

12. E. Koch, Wirkungsbereichpolyeder und Wirkungsbereichsteilungen zu kubischen Gitterkomplexes mit weniger als drei Freiheitsgraden, Ph. D. Thesis, Philipps-Universität Marburg/Lahn, 1972.

13. E. Koch and W. Fischer, Wirkungsbereichstypen einer verzerten Diamantkonfiguration mit Kugelpackungscharakter, Krist. 135 (1972), 73-92.

14. E. H. Lockwood and R. H. Macmillan, Geometric Symmetry, Cambridge University Press, Cambridge, 1978.

15. J. Milnor, Hilbert's 18 problem: on crystallographic groups, fundamental domains, and on sphere packing, in: Proceedings of the Symposium in Pure Mathematics of the American Mathematical Society, Northern Illinois University, Dekalb, Illinois, 1974, pp. 491-507.

16. W. Novacki, Homogene Raumteilung und Kristallstruktur, Ph. D. Thesis, E. T. H. Zürich, 1935.

17. D. Schattschneider, The plane symmetry groups: their recognition and notation, Amer. Math. Monthly 85 (1978), 439-450.

18. D. Schattschneider and M. Senechal, Tilings, in: Handbook of Discrete and Computational Geometry (J. E. Goodman and J. O'Rourke, eds.), CRC Press, Boca Raton, FL, 1997, pp. 43-63.

19. F. W. Smith, The structure of aggregates-a class of 20-faced space-filling polyhedra, Canad. J. Phys. 43 (1965), 2052-2055.

Received October 4, 1999, and in revised form May 30, 2000. Online publication December 4, 2000. 